\title{
Circulating extracellular vesicle-encapsulated microRNA as screening biomarkers for intraductal papillary mucinous neoplasm
}

\author{
YUKI SATO, REI SUZUKI, TADAYUKI TAKAGI, MITSURU SUGIMOTO and HIROMASA OHIRA \\ Department of Gastroenterology, Fukushima Medical University School of Medicine, Fukushima 960-1295, Japan
}

Received May 23, 2020; Accepted September 17, 2020

DOI: $10.3892 / 01.2020 .12178$

\begin{abstract}
Since intraductal papillary mucinous neoplasms (IPMNs) occasionally contain pancreatic malignancies, it is vital to develop a screening program that can detect IPMNs in the general population and that can identify IPMNs with high malignant potential. The present study investigated whether microRNAs (miRNAs/miRs) in the blood may be diagnostic markers for IPMN screening. Initially, extracellular vesicle-encapsulated miRNAs (EV-miRNAs) in the serum with altered expression between IPMN, IPMN-derived carcinoma (IPMC) and control samples, were identified using microarray analysis. To validate the microarray results, the expression levels of selected EV-miRNAs were detected. Briefly, serum EV-miRNAs were extracted from 38 patients with IPMN (11 patients with IPMC and 27 patients with benign IPMN) and 21 non-tumor controls. The results of the microarray analysis revealed that the expression levels of EV-miR-22-3p, EV-miR-4539 and EV-miR-6132 were higher in the IPMN and IPMC serum samples compared with those in the control samples. With regards to discriminating IPMNs from controls, only miR-4539 exhibited a significant difference $(\mathrm{P}=0.004)$. In the comparison between IPMN and IPMC, carcinogenic antigen 19-9 (CA19-9) and EV-miR-6132 exhibited significant differences $(\mathrm{P}=0.01$ and $\mathrm{P}=0.007$, respectively). Receiver operating characteristic (ROC) curve analysis demonstrated that EV-miR-4539 could discriminate patients with IPMNs from control patients, with an area under the curve (AUC) of 0.72 . Additionally, ROC analysis indicated that the markers could discriminate patients with IPMC from benign IPMN, with AUC values of 0.77 for EV-miR-6132 and 0.74 for CA19-9. In conclusion, the present study suggested that EV-miRNAs may be used as diagnostic markers for the
\end{abstract}

Correspondence to: Dr Rei Suzuki, Department of Gastroenterology, Fukushima Medical University School of Medicine, 1 Hikarigaoka, Fukushima 960-1295, Japan

E-mail: subaru@fmu.ac.jp

Key words: intraductal papillary mucinous neoplasm, intraductal papillary mucinous neoplasm-derived carcinoma, extracellular vesicle, microRNA, screening detection of IPMNs in the general population as well as for identifying IPMNs with high malignant potential.

\section{Introduction}

Intraductal papillary mucinous neoplasms (IPMNs) are epithelial neoplasms composed of mucin-producing columnar cells, and IPMNs are classified as 2 types: Main-duct type (MD-IPMN) and branch-duct type IPMN (BD-IPMN). Since IPMN occasionally develops into malignancy (IPMN-derived carcinoma: IPMC) (MD-IPMN: Range, 36-100\%, BD-IPMN: Range, 6.3-46.5\%), we need to develop a screening strategy that can detect IPMN in the general population and identify IPMC $(1,2)$. For a reliable screening program, establishment of diagnostic tests with low invasiveness, high diagnostic yield and high reproducibility is essential. In this context, biomarkers from body fluids can play an important role in IPMN screening.

A considerable number of biomarker studies have evaluated markers for use in detecting IPMCs among IPMN patients (3-6). Carcinogenic antigen 19-9 (CA19-9) is the only biomarker described in the guidelines; however, a recent meta-analysis reported its diagnostic performance as highly specific but not sensitive in identifying IPMC in IPMNs (7). Moreover, no biomarker has demonstrated reliable diagnostic ability in detecting IPMNs in the general population. To overcome this challenge, we need to establish new biomarkers for IPMN screening.

Circulating microRNAs (miRNAs) have been shown to have diagnostic potential as blood biomarkers for various tumors (8-11). miRNAs are small, noncoding RNAs (18-25 nucleotides in length) that regulate gene expression at the posttranscriptional level by promoting the degradation of messenger RNAs or by blocking messenger RNA translation. They can be loaded into exosomes or other extracellular vesicles (extracellular vesicle-encapsulated miRNA: EV-miRNA) or exist freely in the blood circulation (circulating miRNA). Among these two forms, EV-miRNA has several advantages over circulating miRNA: i) It can be specifically released for cell-to-cell communication, and ii) lipid membrane coverage protects miRNA from RNase degradation (12).

In this study, we aimed to determine whether serum EV-miRNAs could be diagnostic markers of IPMNs in the general population. In addition, the diagnostic yield of 
serum EV-miRNAs in distinguishing IPMNs and IPMC was evaluated.

\section{Materials and methods}

Patients and treatments. First, we selected 4 patients with IPMNs ( 2 benign IPMNs and 2 IPMCs) and 4 controls without any type of tumor for microarray analysis to identify differences in the gene expression of serum EV-miRNAs between these groups. To validate the microarray results, we enrolled 38 consecutive patients who were diagnosed with IPMNs by imaging modalities at Fukushima Medical University Hospital and 21 patients without any neoplastic lesions as controls between June 2015 and November 2019. The diagnostic criteria were as follows: i) Dilation of the MD and/or a cystic dilation of the BD, and ii) secretion of mucin from the major or minor papilla identified by endoscopic retrograde cholangiopancreatography or duodenoscopy.

Clinical data including age, sex, background diseases in controls, presence of symptoms (jaundice, body weight loss, etc.), history of diabetes mellitus, pancreatitis, smoking, family history of pancreatic tumor, and serum tumor markers, including carcinoembryonic antigen (CEA) and CA 19-9, were retrieved from electronic medical records. Patients with co-existing tumors or active infection (i.e., cholangitis or cholecystitis) were excluded from the analysis. All recruited patients were evaluated with CT or MRI, and the location of the lesion, maximum diameter of cyst and main pancreatic duct (MPD), and the presence of mural nodules were determined. Medical management of the disease was also evaluated. Classification of BD-IPMN and others was performed according to the 2017 International Consensus Guideline (1).

The study protocol conformed to the ethical guidelines of the 1975 Declaration of Helsinki and was approved by the institutional review board of Fukushima Medical University (IRB \#2387). All participants provided written informed consent.

Sample collection. To obtain the serum samples, $8 \mathrm{ml}$ of blood was collected and incubated at room temperature for at least $60 \mathrm{~min}$ to allow clotting. Samples were then centrifuged at $1,000 \mathrm{xg}$ for $10 \mathrm{~min}$. The serum was collected and stored in aliquots at $-80^{\circ} \mathrm{C}$.

miRNA preparation and microarrays. Serum miRNA was extracted from serum using the exoRNeasy Serum/Plasma Midi kit (Qiagen) according to the manufacturer's protocol. RNA quantity and quality were determined using an Agilent Bioanalyzer (Agilent Technologies), as recommended. miRNA microarrays were manufactured by Agilent Technologies. Briefly, RNA was dephosphorylated using calf intestinal alkaline phosphatase (CIP) master mix incubated at $37^{\circ} \mathrm{C}$ for $30 \mathrm{~min}$. Dephosphorylated RNA was denatured with DMSO incubated at $100^{\circ} \mathrm{C}$ for $5 \mathrm{~min}$ and then immediately transferred to ice for $2 \mathrm{~min}$. These products were mixed with a ligation master mix for T4 RNA ligase and $\mathrm{Cy} 3-\mathrm{pCp}$ (Cyanine 3-Cytidine biphosphate) and incubated at $16^{\circ} \mathrm{C}$ for $2 \mathrm{~h}$. Labeled RNA was dried using a vacuum concentrator at $55^{\circ} \mathrm{C}$ for $1.5 \mathrm{~h}$. Cy3-pCp-labeled RNA was hybridized on an Agilent SurePrint G3 Human miRNA 8x60K Rel.21
Table I. Background disease of patients in microRNA array analysis.

\begin{tabular}{llc}
\hline Sample nos. & Disease & Group name \\
\hline $1-4$ & Control & A1 \\
5 and 6 & IPMC & A2 \\
7 and 8 & IPMN & A3 \\
\hline
\end{tabular}

IPMN, intraductal papillary mucinous neoplasm; IPMC, IPMN-derived carcinoma.

(design ID: 070156) array at $55^{\circ} \mathrm{C}$ for $20 \mathrm{~h}$. After washing, microarrays were scanned using an Agilent SureScan Microarray Scanner System (G4900DA). The intensity values of each scanned feature were quantified using Agilent Feature Extraction software version 12.1.1.1, which performs background subtractions. We only used features that were flagged as no errors (detected flags) and excluded features that were not positive, not significant, not uniform, not above background, saturated, and population outliers (not detected flags). These expression analyses were performed with Agilent GeneSpring GX version 14.9.1.

DigitalPCR.Digital PCR and quantification of the absolutelevels of serum miRNAs were performed using the Quant-Studio 3D Digital PCR system (Thermo Fisher Scientific, Inc.). Data were analyzed using QuantStudio 3D Analysis Suite Cloud Software (Thermo Fisher Scientificc, Inc.). The digital PCR mixture contained $5.0 \mu 1$ of the RT product, $1.0 \mu \mathrm{l}$ of nuclease-free $\mathrm{H}_{2} \mathrm{O}$, $7.50 \mu 1$ of the QuantStudio ${ }^{\mathrm{TM}}$ 3D Digital PCR Master Mix, and $0.75 \mu \mathrm{l}$ of the TaqMan MiRNA Assay-1 (20X) for let-7d. Samples were individually loaded onto the QuantStudio 3D digital PCR 20K chip kit v2 using the QuantStudio 3D digital PCR Chip Loader. Digital PCR was performed in a Proflex 2X flat block thermal cycler (Applied Biosystems) using standard conditions: $96^{\circ} \mathrm{C}$ for 10 min followed by 39 cycles of $60^{\circ} \mathrm{C}$ for $2 \mathrm{~min}, 98^{\circ} \mathrm{C}$ for $30 \mathrm{sec}$, and $60^{\circ} \mathrm{C}$ for $2 \mathrm{~min}$. Chips were read on the QuantStudio 3D digital PCR instrument, and the number of FAM-positive and FAM-negative (empty) wells was quantified $(13,14)$.

Target gene prediction and pathway enrichment analyses. Target gene prediction was performed using DIANA-miRPath software (15). Specifically, we investigated whether tumor suppressor genes were targeted by these 3 miRNAs because aberrant tumor suppressor gene methylation was observed in malignant IPMN (16).

To predict cell signaling pathways that were potentially influenced by the EV-miRNAs, Kyoto Encyclopedia of Genes and Genomes (KEGG) pathway enrichment analyses were performed via the Database for Annotation, Visualization and Integrated Discovery (DAVID 6.8; http://david.abcc.ncifcrf. gov/) $(17,18)$. KEGG pathways with a P-value $<0.05$ were considered significantly enriched.

Statistical analysis. Continuous variables (i.e., age, cyst size, serum CEA and CA 19-9 levels and GS) are reported as the 
Table II. Different expressions of miRNAs between each group.

\begin{tabular}{|c|c|c|c|c|c|c|}
\hline Systematic name & $\begin{array}{c}\text { FC } \\
{[(\mathrm{A} 2) \mathrm{vs} .(\mathrm{A} 3)]}\end{array}$ & $\begin{array}{c}\log \mathrm{FC} \\
{[(\mathrm{A} 2) \mathrm{vs} .(\mathrm{A} 3)]}\end{array}$ & $\begin{array}{c}\text { FC } \\
{[(\mathrm{A} 2) \mathrm{vs} .(\mathrm{A} 1)]}\end{array}$ & $\begin{array}{c}\log \mathrm{FC} \\
{[(\mathrm{A} 2) \mathrm{vs} .(\mathrm{A} 1)]}\end{array}$ & $\begin{array}{c}\mathrm{FC} \\
{[(\mathrm{A} 3) \mathrm{vs} .(\mathrm{A} 1)]}\end{array}$ & $\begin{array}{c}\log \mathrm{FC} \\
{[(\mathrm{A} 3) \mathrm{vs} .(\mathrm{A} 1)]}\end{array}$ \\
\hline hsa-miR-6132 & 4.55 & 2.19 & 21.55 & 4.43 & 4.74 & 2.24 \\
\hline hsa-miR-22-3p & 2.92 & 1.55 & 7.54 & 2.91 & 2.58 & 1.37 \\
\hline hsa-miR-4539 & 2.94 & 1.56 & 7.54 & 2.91 & 2.56 & 1.36 \\
\hline hsa-miR-6732-3p & 9.31 & 3.22 & 7.54 & 2.91 & -1.23 & -0.30 \\
\hline hsa-miR-4534 & 2.16 & 1.11 & 2.30 & 1.20 & 1.06 & 0.09 \\
\hline hsa-miR-3679-5p & 1.83 & 0.87 & 1.60 & 0.67 & -1.15 & -0.20 \\
\hline
\end{tabular}

miR/miRNA, microRNA; FC, fold change.

median and range and were compared using Mann-Whitney analysis. Categorical variables (i.e., sex and location of disease) were analyzed using Fisher's exact test. The diagnostic yield of CEA and CA 19-9 levels and EV-miRNAs in distinguishing whole IPMN (benign IPMN and IPMC) from the control and IPMC from benign IPMN was assessed using the area under the receiver operating characteristic (ROC) curve. Statistical analyses were performed using SPSS version 26.0 for Windows (IBM Corp.), and figures were generated with Prism 7 (GraphPad Software, Inc.). $\mathrm{P}<0.05$ was considered to indicate statistical significance.

\section{Results}

Identification of miRNAs for IPMN screening. We first identified miRNAs showing altered expression in 2 IPMNs, 2 IPMCs and 4 controls (Table I). Among the 2588 mature miRNAs evaluated in the microarray, we found $3 \mathrm{EV}$-miRNAs [hsa-miR-6132 (EV-miR-6132), hsa-miR-22-3p (EV-miR-22) and hsa-miR-4539 (EV-miR-4539)] that could discriminate IPMN, IPMC and control (Table II). As shown in Fig. 1, target prediction using DIANA-miRPath software revealed that these 3 miRNAs could target various genes. Regarding tumor suppressor genes, we found that miR-22-3p could target TP53INP1 and mir-6132 could target 3 genes (tuberous sclerosis complex 2: TSC2, tumor protein p53-inducible protein 11: TP53I11 and protein phosphatase 2 regulatory subunit B'Delta: PPP2R5D).

In addition, pathway enrichment analyses found that these 3 miRNAs could influence the NOD-like receptor signaling pathway (6 target genes involved), glycosphingolipid biosynthesis-lacto and neolacto series-(1 target gene involved) and fat digestion and absorption (5 target genes involved) (Table III).

Clinical characteristics of the patients. To validate whether the 3 miRNAs could be diagnostic markers for IPMN, we conducted a validation study using digital PCR. The clinical characteristics of patients in the IPMN $(n=38)$ and control $(n=21)$ groups are presented in Table IV. Briefly, there were no significant differences in age, sex, absence of symptoms, history of diabetes, pancreatitis, smoking, or family history of pancreatic cancer between the IPMN group and the control group. With regard to management, a total of 9 patients underwent surgery among 38 IPMN patients. We confirmed

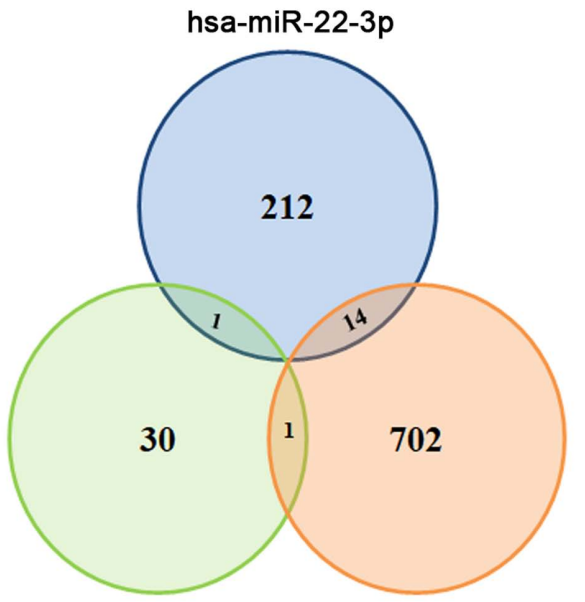

hsa-miR-4539

hsa-miR-6132

Figure 1. Selection of candidate EV-miRNAs for identifying benign IPMNs and IPMC. Target gene prediction using DIANA-miRPath software revealed that EV-miR-22-3p could target 227 genes, EV-miR-4539 could target 32 genes and EV-miR-6132 could target 717 genes. Moreover, several genes were targeted by 2 EV-miRNAs. EV, extracellular vesicle; miRNA/miR, microRNA; IPMN, intraductal papillary mucinous neoplasm; IPMC, IPMN-derived carcinoma.

the pathological diagnosis of the resected cases according to the latest classification (1): Low-grade dysplasia $(n=4)$, high-grade dysplasia $(n=1)$, and invasive carcinoma $(n=4)$. Twenty-eight patients did not require surgery and were continuously observed. One patient was treated with chemotherapy.

We also divided IPMNs into 2 groups, namely, IPMC $(n=11)$ and benign IPMN without any suspicious findings of malignancy $(n=27)$, and compared clinical characteristics as shown in Table V. Clinical symptoms were observed more frequently in IPMC than IPMN (36.3 vs. 0.0\%, P=0.0001). Additionally, the median diameter of the MPD was significantly larger in IPMC than in IPMN (11.3 vs. $4.4 \mathrm{~mm}$, $\mathrm{P}=0.0003)$.

miRNAs as diagnostic markers for IPMN. Fig. 2 shows the differences in each biomarker between the control and IPMN (both benign IPMN or IPMC) groups (Fig. 2) and between benign IPMN and IPMC groups (Fig. 3). With regard to discriminating IPMNs from control, only EV-miR-4539 
Table III. Results of KEGG pathway analysis.

\begin{tabular}{lcl}
\hline KEGG pathways & P-value & \multicolumn{1}{c}{ Involved genes } \\
\hline $\begin{array}{l}\text { KEGG pathway: miR-22-3p and miR-6132 } \\
\text { NOD-like receptor signaling pathway }\end{array}$ & 0.04 & $\begin{array}{l}\text { NF-kappa-B inhibitor beta (NFKBIB), Caspase 1 (CASP1), } \\
\text { NACHT, LRR and PYD domains-containing protein 3 (NLRP3), } \\
\text { mitogen-activated protein kinase 11 (MAPK11), Suppressor } \\
\text { of G2 allele of SKP1 homolog (SUGT1), mitogen-activated } \\
\text { protein kinase 1 (MAPK1) }\end{array}$
\end{tabular}

KEGG pathway: miR-22-3p and miR-4539

Glycosphingolipid biosynthesis-lacto and

$0.002 \quad$ Fucosyltransferase 9 (FUT9)

neolacto series

KEGG pathway: miR-4539 and miR-6132

Fat digestion and absorption

$0.04 \quad$ Fatty acid binding protein 1 (FABP1), Apolipoprotein A4

(APOA4), CD36, microsomal triglyceride transfer protein

(MTTP), Phospholipase A2 Group IIC (PLA2G2C)

KEGG, Kyoto Encyclopedia of Genes and Genomes; miR, microRNA.

Table IV. Clinical characteristics of patients with IPMN and controls.

\begin{tabular}{|c|c|c|c|}
\hline Characteristics & Control $(n=21)$ & IPMN (n=38) & P-value \\
\hline Median age, years (range) & $71.0(46.0-89.0)$ & $74.0(47.0-91.0)$ & 0.51 \\
\hline Sex, male $(\%)$ & $13(61.9)$ & $19(50.0)$ & 0.42 \\
\hline \multicolumn{4}{|l|}{ Background disease } \\
\hline Bile duct stone, $\mathrm{n}(\%)$ & $14(66.7)$ & & \\
\hline Chronic pancreatitis, n (\%) & $7(33.3)$ & & \\
\hline Presence of symptoms, n (\%) & $3(14.2)$ & $7(18.4)$ & 0.99 \\
\hline Hx of diabetes mellitus, $\mathrm{n}(\%)$ & $4(19.0)$ & $11(28.9)$ & 0.53 \\
\hline Hx of pancreatitis, n (\%) & $2(9.5)$ & $2(5.3)$ & 0.61 \\
\hline Hx of smoking, never/ever/current & $9 / 6 / 6$ & $19 / 9 / 10$ & 0.29 \\
\hline Family Hx of pancreatic tumor (\%) & $0 \quad(0.0)$ & $2(5.3)$ & 0.53 \\
\hline IPMN subtype, branch-duct, n (\%) & & $25(65.8)$ & \\
\hline IPMC, n (\%) & & $11(28.9)$ & \\
\hline \multicolumn{4}{|l|}{ Management, n (\%) } \\
\hline Follow-up & $21(100.0)$ & $28(73.6)$ & \\
\hline Surgical resection & & $9(23.6)$ & \\
\hline Chemotherapy & & $1 \quad(2.6)$ & \\
\hline
\end{tabular}

Hx, history; IPMN, intraductal papillary mucinous neoplasm; IPMC, IPMN-derived carcinoma.

showed a significant difference $(\mathrm{P}=0.004)$ (Fig. 2A). ROC analysis showed that 5 markers could discriminate patients with IPMN and from control patients, with areas under the curve (AUCs) of 0.72 for EV-miR-4539 (95\% confidence interval [CI]: $0.59-0.85), 0.55$ for CEA (95\% CI, 0.39-0.71), 0.55 for CA19-9 (95\% CI, 0.41-0.71), 0.59 for EV-miR-22 (95\% CI, 0.42-0.76) and 0.64 for EV-miR-6132 (95\% CI, 0.47-0.81). As shown in Fig. 2B, EV-miR-4539 had the highest diagnostic yield compared with other markers at the cutoff value of $3.2 \mathrm{copies} / \mu 1$, and the sensitivity and specificity were 60.5 and $95.2 \%$, respectively (Fig. 2B).
In the comparison between benign IPMN and IPMC, CA19-9 and EV-miR-6132 showed significant differences $(\mathrm{P}=0.01$ and 0.007 , respectively) (Fig. 3A). ROC analysis showed that the markers could discriminate patients with IPMC from patients with benign IPMN, with an AUC of 0.77 for EV-miR-6132 (95\% CI, 0.61-0.93), 0.74 for CA19-9 (95\% CI, 0.52-0.97), 0.61 for EV-miR-22 (95\% CI, 0.41-0.81), 0.58 for EV-miR-4539 (95\% CI, 0.40-0.77) and 0.55 for CEA (95\% CI, 0.34-0.77). EV-miR-6132 showed the highest diagnostic yield compared with other markers at the cutoff value of $1.4 \mathrm{copies} / \mu 1$ and the sensitivity and specificity were 88.3 and $65.4 \%$, respectively (Fig. 3B). 
Table V. Clinical characteristics of patients with IPMC and IPMN.

\begin{tabular}{|c|c|c|c|}
\hline Characteristics & Benign IPMN (n=27) & $\operatorname{IPMC}(\mathrm{n}=11)$ & P-value \\
\hline Median age, years (range) & $69.5(48.0-89.0)$ & $74.5(47.0-91.0)$ & 0.71 \\
\hline Sex, male $(\%)$ & $11(40.7)$ & $8(63.6)$ & 0.15 \\
\hline Presence of symptoms, n (\%) & $0 \quad(0.0)$ & $7(36.3)$ & 0.0001 \\
\hline Hx of diabetes mellitus, $\mathrm{n}(\%)$ & $7(33.3)$ & $4(33.3)$ & 0.99 \\
\hline Hx of pancreatitis, $\mathrm{n}(\%)$ & $2(7.4)$ & $0(0.0)$ & 0.99 \\
\hline Hx of smoking, never/ever/current & $15 / 5 / 7$ & $5 / 5 / 1$ & 0.29 \\
\hline Family $\mathrm{Hx}$ of pancreatic tumor (\%) & $1 \quad(3.7)$ & $1(9.1)$ & 0.50 \\
\hline Location of the lesion, $\mathrm{Ph} / \mathrm{Pb} / \mathrm{Pt} /$ diffuse & $12 / 7 / 4 / 4$ & $6 / 3 / 0 / 2$ & 0.60 \\
\hline Median cyst diameter, mm (range) & $24.5(12.0-63.0)$ & $31.0(10.0-170.0)$ & 0.09 \\
\hline Median MPD diameter, mm (range) & $4.4(1.5-13.0)$ & $11.2(2.2-33.0)$ & 0.0003 \\
\hline Median size of mural nodule, mm (range) & $8.1(3.0-15.0)$ & $18.0(4.0-50)$ & 0.02 \\
\hline
\end{tabular}

$\mathrm{Hx}$, history; $\mathrm{Ph} / \mathrm{Pb} / \mathrm{Pt}$, pancreatic head, body and tail; MPD, main pancreatic duct; IPMN, intraductal papillary mucinous neoplasm; IPMC, IPMN-derived carcinoma.

A

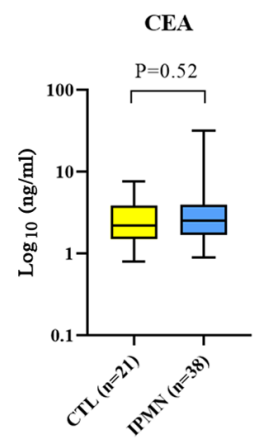

EV-miR-22

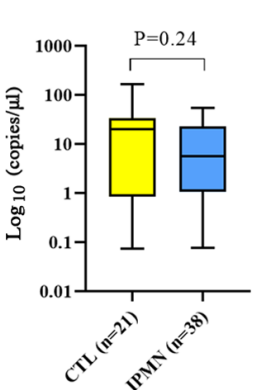

EV-miR-4539

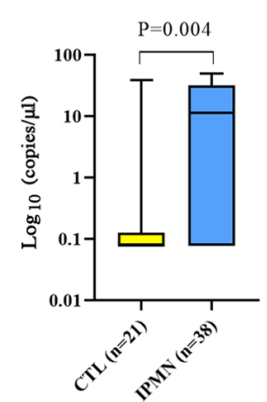

EV-miR-6132

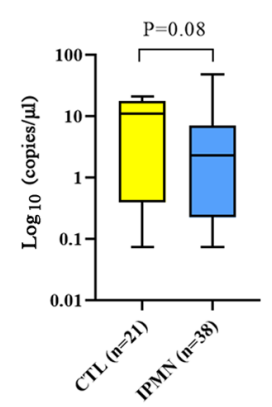

B

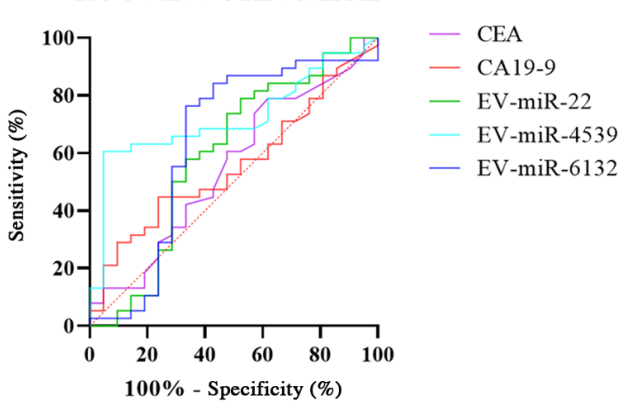

\begin{tabular}{|c|c|c|c|c|c|}
\hline \multicolumn{2}{|l|}{ Markers } & \multirow{2}{*}{$\begin{array}{c}\text { Cut-off value } \\
1.6\end{array}$} & \multirow{2}{*}{$\begin{array}{c}\text { AUC } \\
0.55\end{array}$} & \multirow{2}{*}{$\begin{array}{c}\text { Sensitivity (\%) } \\
78.9\end{array}$} & \multirow{2}{*}{$\begin{array}{c}\text { Specificity (\%) } \\
38.1\end{array}$} \\
\hline CEA $\quad(\mathrm{ng} / \mathrm{ml}$ & & & & & \\
\hline CA19-9 (U/ml & & 14.6 & 0.55 & 44.7 & 76.2 \\
\hline EV-miR-22 & (copies $/ \mu \mathrm{l})$ & 24.2 & 0.59 & 78.9 & 47.6 \\
\hline EV-miR-4539 & (copies/ $/ \mu \mathrm{l})$ & 3.2 & 0.72 & 60.5 & 95.2 \\
\hline EV-miR-6132 & (copies/ $/ \mu \mathrm{l}$ ) & 6.8 & 0.64 & 76.2 & 66.7 \\
\hline
\end{tabular}

Figure 2. Comparison of serum biomarkers between the control and IPMN groups. (A) Only EV-miR-4539 showed a statistically significant difference. (B) EV-miR-4539 showed the highest diagnostic yield among the markers. Data were presented in box-and-whisker plot and error bars in figures were presented with range (minimum to maximum). EV, extracellular vesicle; miRNA/miR, microRNA; IPMN, intraductal papillary mucinous neoplasm; CEA, carcinoembryonic antigen; CA19-9, carcinogenic antigen 19-9; ROC, receiver operating characteristic; AUC, area under the curve.

With regard to the serum levels of biomarkers and the existence of high-risk indicators, the serum level of CA19-9 was higher in patients with obstructive jaundice (Fig. 4). The serum level of EV-miR-6132 was higher in patients with high-risk indicators (mural nodules and MPD diameter more than $10 \mathrm{~mm}$ ) than in patients without them.

\section{Discussion}

In the present study, we aimed to confirm the potentially useful EV-miRNAs (EV-miR-22, EV-miR-4539 and EV-miR-6132) for IPMN and IPMC detection in microarray analysis. To the best of our knowledge, this is the first study that suggested the usefulness of EV-miRNAs in IPMN screening. It was found that EV-miR-4539 was better at distinguishing IPMNs from non-tumor controls than other biomarkers. While the diagnostic yield of EV-miR-6132 to discriminate IPMNs and IPMC was comparable to that of CA19-9, it had the advantage that the expression level was not influenced by biliary obstruction.

miRNAs can play multifunctional roles in cancer progression. With regard to IPMN, Habbe et al first reported abnormal 
A
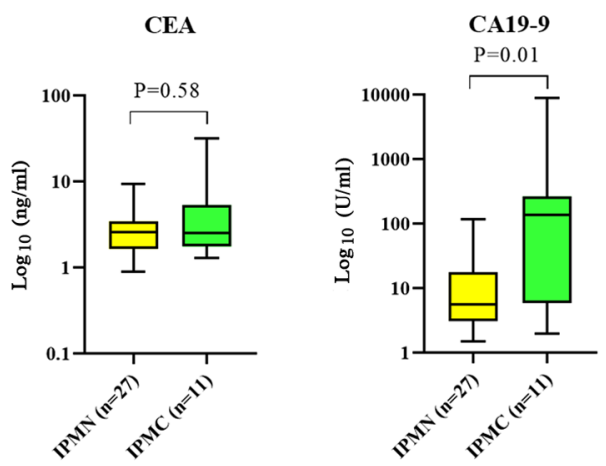

B
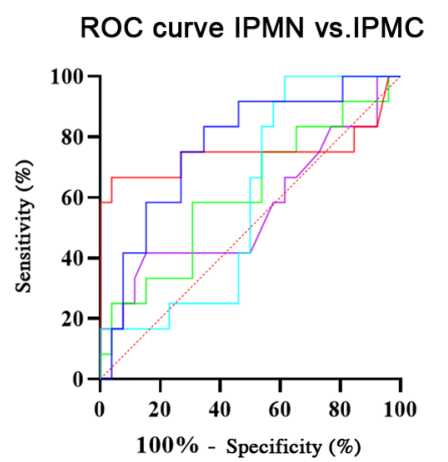

- CEA

- CA19-9

- EV-miR-22

EV-miR-4539

- EV-miR-6132

\begin{tabular}{|c|c|c|c|c|}
\hline Markers & Cut-off value & AUC & Sensitivity (\%) & Specificity (\%) \\
\hline CEA $\quad(\mathrm{ng} / \mathrm{ml})$ & 4.0 & 0.55 & 41.6 & 84.2 \\
\hline CA19-9 (U/ml) & 63.8 & 0.74 & 66.7 & 96.1 \\
\hline EV-miR-22 $\quad($ copies/ $/ \mathrm{l})$ & 12.7 & 0.61 & 58.3 & 69.2 \\
\hline EV-miR-4539 (copies $/ \mu \mathrm{l})$ & 30.9 & 0.58 & 100.0 & 38.4 \\
\hline EV-miR-6132 (copies $/ \mu l)$ & 1.4 & 0.77 & 88.3 & 65.4 \\
\hline
\end{tabular}

EV-miR-4539

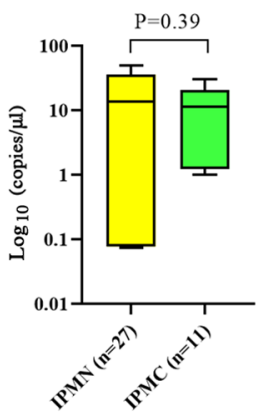

EV-miR-6132

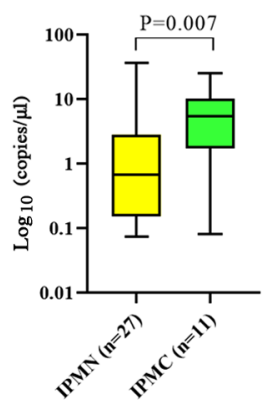

Figure 3. Comparison of serum biomarkers between benign IPMN and IPMC groups. (A) CA19-9 EV-miR-6132 showed a significant difference. (B) miR-6132 had the highest diagnostic yield among the markers. Data were presented in box-and-whisker plot and error bars in figures were presented with range (minimum to maximum). EV, extracellular vesicle; miRNA/miR, microRNA; IPMN, intraductal papillary mucinous neoplasm; IPMC, IPMN-derived carcinoma; CEA, carcinoembryonic antigen; CA19-9, carcinogenic antigen 19-9; ROC, receiver operating characteristic; AUC, area under the curve.

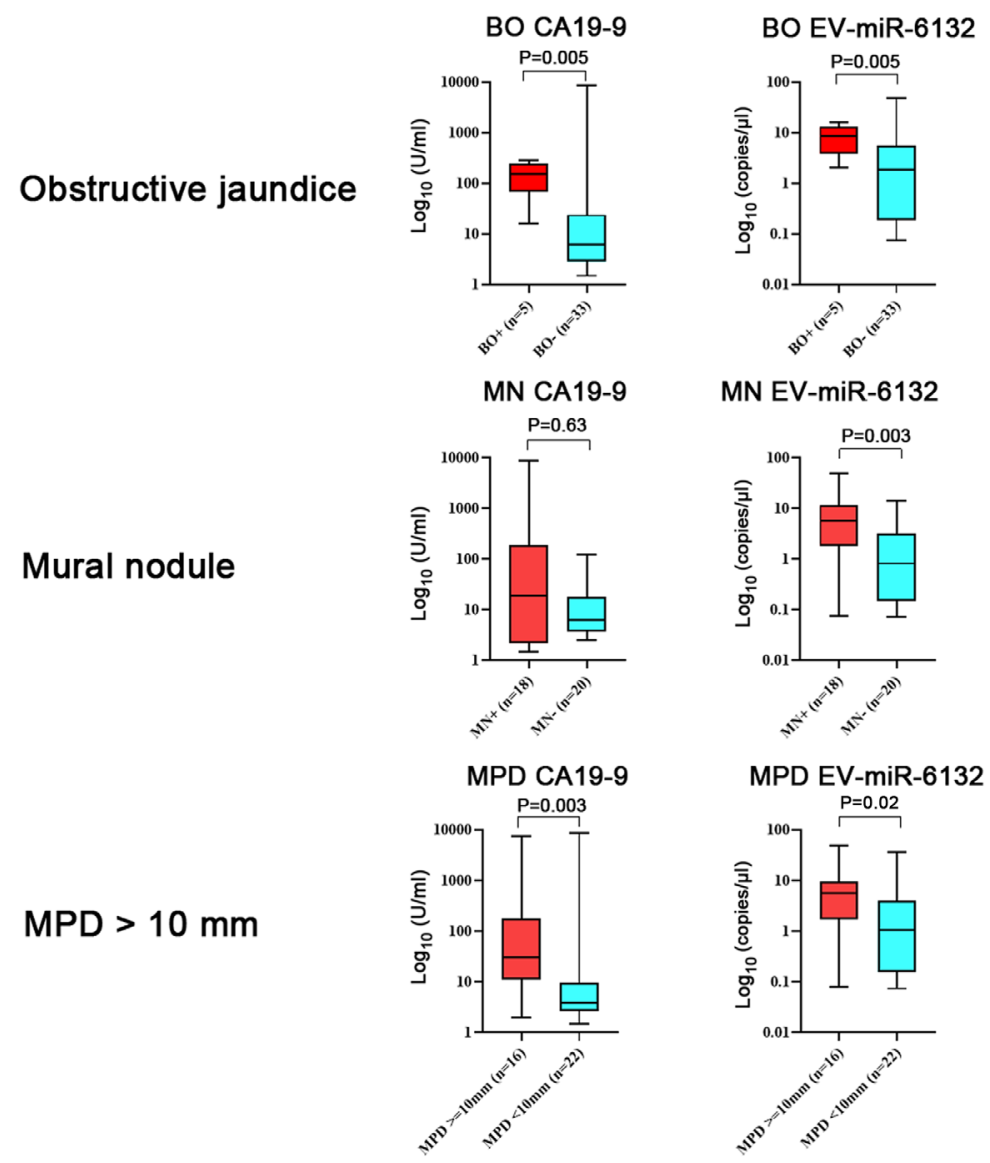

Figure 4. Expression levels of biomarkers per high-risk indicator. CA19-9 was highly expressed in patients with $\mathrm{BO}(\mathrm{P}=0.005)$. Data were presented in box-and-whisker plot and error bars in figures were presented with range (minimum to maximum). CA19-9, carcinogenic antigen 19-91 EV, extracellular vesicle; miRNA/miR, microRNA; BO, biliary obstruction; MN, mural nodule; MPD, main pancreatic duct. 
miRNA expression in IPMN (19). In this study, the expression of miR-155 was found to be elevated in both IPMN tissue and pancreatic juice. Authors suggested that miRNA could be a diagnostic marker for pancreatic neoplasms. Subsequently, multiple studies focused on the identification of high-risk IPMN, and the results suggested that many miRNAs in the blood, pancreatic juice, and cystic fluid could be useful diagnostic markers. More recently, some researchers have attracted the attention of miRNAs encapsulated in EV-like exosomes since they are supposed to be stabilized to avoid degradation in the blood and highly enriched compared to circulating miRNAs. Goto et al (20) compared the diagnostic yield of serum circulating miRNAs and exosomal miRNAs in distinguishing control tissues from IPMNs. Their study was quite informative as the diagnostic yield of exosomal miRNAs was found to be $5-20 \%$ superior to that of serum circulating miRNAs (e.g., exosomal miR-21: AUC 0.826, accuracy 80\%). Circulating miRNA-21: AUC 0.653, accuracy 62.3\%). Although they did not focus on discriminating IPMCs from benign IPMNs, in contrast to our study, the results indicated the superiority of encapsulated miRNAs over circulating miRNAs as diagnostic markers.

While we cannot find any relevant data regarding miR-6132 and tumors, the expression of miR-22 and miR-4539 in cancer tissue has been studied in gastric cancer, rhabdomyosarcoma, breast cancer, prostate cancer, osteosarcoma and papillary thyroid cancer (21-30). Most studies have reported that, in contrast to their expression levels in the blood, the expression levels of these miRNAs are decreased in cancer tissue. Moreover, in vivo and in vitro studies regarding the biophysical properties of those miRNAs encapsulated in EVs have not been conducted. Hence, the mechanism by which the 3 EV-miRNAs in the blood contribute to IPMN progression has not been elucidated. As in silico analysis suggests, inhibition of the NOD-like receptor signaling pathway may be a cause of IPMN progression. NOD-like receptors are genetically conserved proteins that belong to the cellular pattern recognition receptor protein family. They are important components in the innate immune system of mammals, regulating the immune response and inflammatory response. Recently, accumulative evidence has extended the concept that the NOD-like receptor signaling pathway contributes to the activation of the antitumor immune response by priming antitumor $\mathrm{CD}^{+}$and $\mathrm{CD}^{+} \mathrm{T}$ cells (31). Considering this result, increased expression of EV-miRNAs might be attributable to suppression of the antitumor immune response and progression of IPMN. Regarding the other 2 pathways, glycosphingolipid biosynthesis-lacto and neolacto series- and fat digestion and absorption, we could not find any relevant studies.

Regarding progression of IPMN to IPMC, EV-miR-6132 may play an important role because its serum levels are higher in IPMC patients than in benign IPMN patients. Further evaluation revealed that 3 tumor suppressor genes (TSC2, TP53I11 and PPP2R5D) were found among 717 target genes. Aberrant tumor suppressor gene methylation which lead to gene suppression just like miRNA is often found in IPMC, and we speculate that the high level of EV-miR-6132 in the serum of IPMC patients may influence tumor suppressor gene activity as well (16).
Several limitations were found in this study. First, this study was conducted in a single referral center, and the results may not be generalizable to all patients with IPMNs and IPMCs. The relatively small sample size also limited the reliability of our statistical analysis. Second, only 9 of 38 IPMN patients underwent surgical resection. The remaining 29 patients were diagnosed with a benign IPMN or an IPMC based on the imaging findings and clinical outcomes. Third, we did not investigate the expression levels of the 3 miRNAs in the tumor tissue, and we do not know whether the miRNAs directly contribute to carcinogenesis or tumor progression. Therefore, we must conduct a further study that includes a large number of patients with histopathological evaluation.

In conclusion, we found that EV-miRNAs can be diagnostic markers for use in detecting IPMNs in the general population as well as in identifying IPMNs with high malignant potential.

\section{Acknowledgements}

The authors would like to thank Ms. Chikako Sato and Ms. Rie Hikichi (Department of Gastroenterology, Fukushima Medical University School of Medicine) for their assistance in the experiments.

\section{Funding}

No funding was received.

\section{Availability of data and materials}

All data generated or analyzed during the present study are included in the published article.

\section{Authors' contributions}

YS, RS and HO conceived and designed the experiments. YS and RS performed the experiments. YS, RS, TT, MS and HO collected and analyzed the data. YS, RS, TT, MS and HO interpreted the results and wrote the manuscript. All authors read and approved the manuscript, and agree to be accountable for all aspects of the research and to guarantee for the accuracy and integrity of any part of the work.

\section{Ethics approval and consent to participate}

The study protocol conformed to the ethical guidelines of the 1975 Declaration of Helsinki and was approved by the institutional review board of Fukushima Medical University (IRB \#2387). All participants provided written informed consent for participation.

\section{Patient consent for publication}

All participants provided written informed consent for publication.

\section{Competing interests}

The authors declare that they have no competing interests. 


\section{References}

1. Tanaka M, Fernandez-Del Castillo C, Kamisawa T, Jang JY Levy P, Ohtsuka T, Salvia R, Shimizu Y, Tada M and Wolfgang CL: Revisions of international consensus Fukuoka guidelines for the management of IPMN of the pancreas Pancreatology 17: 738-753, 2017.

2. Oyama H, Tada M, Takagi K, Tateishi K, Hamada T, Nakai Y, Hakuta R, Ijichi H, Ishigaki $\mathrm{K}$, Kanai $\mathrm{S}$, et al: Long-term risk of malignancy in branch-duct intraductal papillary mucinous neoplasms. Gastroenterology 158: 226-237 e5, 2020.

3. Al-Haddad M, DeWitt J, Sherman S, Schmidt CM, LeBlanc JK, McHenry L, Coté G, El Chafic AH, Luz L, Stuart JS, et al: Performance characteristics of molecular (DNA) analysis for the diagnosis of mucinous pancreatic cysts. Gastrointest Endosc 79: 79-87, 2014.

4. Matthaei H, Wylie D, Lloyd MB, Dal Molin M, Kemppainen J, Mayo SC, Wolfgang CL, Schulick RD, Langfield L, Andruss BF, et al: mRNA biomarkers in cyst fluid augment the diagnosis and management of pancreatic cysts. Clin Cancer Res 18: 4713-4724, 2012.

5. Takano S, Fukasawa M, Maekawa S, Kadokura M, Miura M, Shindo H, Takahashi E, Sato T and Enomoto N: Deep sequencing of cancer-related genes revealed GNAS mutations to be associated with intraductal papillary mucinous neoplasms and its main pancreatic duct dilation. PLoS One 9: e98718, 2014.

6. Moris D, Damaskos C, Spartalis E, Papalampros A Vernadakis S, Dimitroulis D, Griniatsos J, Felekouras E and Nikiteas N: Updates and critical evaluation on novel biomarkers for the malignant progression of intraductal papillary mucinous neoplasms of the pancreas. Anticancer Res 37: 2185-2194, 2017.

7. Tanaka M, Heckler M, Liu B, Heger U, Hackert $T$ and Michalski CW: Cytologic analysis of pancreatic juice increases specificity of detection of malignant IPMN-A systematic review. Clin Gastroenterol Hepatol 17: 2199-2211 e21, 2019.

8. Reddy KB: MicroRNA (miRNA) in cancer. Cancer Cell Int 15: $38,2015$.

9. Peng Y and Croce CM: The role of microRNAs in human cancer. Signal Transduct Target Ther 1: 15004, 2016.

10. Jansson MD and Lund AH: MicroRNA and cancer. Mol Oncol 6: 590-610, 2012.

11. Hayes J, Peruzzi PP and Lawler S: MicroRNAs in cancer: Biomarkers, functions and therapy. Trends Mol Med 20: 460-469, 2014.

12. de Miguel Perez D, Rodriguez Martinez A, Ortigosa Palomo A, Delgado Ureña M, Garcia Puche JL, Robles Remacho A, Exposito Hernandez J, Lorente Acosta JA, Ortega Sánchez FG and Serrano MJ: Extracellular vesicle-miRNAs as liquid biopsy biomarkers for disease identification and prognosis in metastatic colorectal cancer patients. Sci Rep 10: 3974, 2020

13. Conte D, Verri C, Borzi C, Suatoni P, Pastorino U, Sozzi G and Fortunato O: Novel method to detect microRNAs using chip-based QuantStudio 3D digital PCR. BMC Genomics 16: 849, 2015.

14. Suzuki R, Asama H, Waragai Y, Takagi T, Hikichi T, Sugimoto M, Konno N, Watanabe K, Nakamura J, Kikuchi H, et al: Fibrosis-related miRNAs as serum biomarkers for pancreatic ductal adenocarcinoma. Oncotarget 9: 4451-4460, 2017.

15. Vlachos IS, Zagganas K, Paraskevopoulou MD, Georgakilas G, Karagkouni D, Vergoulis T, Dalamagas T and Hatzigeorgiou AG: DIANA-miRPath v3.0: Deciphering microRNA function with experimental support. Nucleic Acids Res 43 (W1): W460-W466, 2015.

16. House MG, Guo M, Iacobuzio-Donahue C and Herman JG: Molecular progression of promoter methylation in intraductal papillary mucinous neoplasms (IPMN) of the pancreas. Carcinogenesis 24: 193-198, 2003.
17. Huangda W,ShermanBT,Zheng X,Yang J,ImamichiT,Stephens R and Lempicki RA: Extracting biological meaning from large gene lists with DAVID. Curr Protoc Bioinformatics Chapter 13: Unit 13 11, 2009. doi: 10.1002/0471250953.bi1311s27.

18. Huang da W, Sherman BT and Lempicki RA: Bioinformatics enrichment tools: Paths toward the comprehensive functional analysis of large gene lists. Nucleic Acids Res 37: 1-13, 2009.

19. Habbe N, Koorstra JB, Mendell JT, Offerhaus GJ, Ryu JK, Feldmann G, Mullendore ME, Goggins MG, Hong SM and Maitra A: MicroRNA miR-155 is a biomarker of early pancreatic neoplasia. Cancer Biol Ther 8: 340-346, 2009.

20. Goto T, Fujiya M, Konishi H, Sasajima J, Fujibayashi S, Hayashi A, Utsumi T, Sato H, Iwama T, Ijiri M, et al: An elevated expression of serum exosomal microRNA-191, -21, -451a of pancreatic neoplasm is considered to be efficient diagnostic marker. BMC Cancer 18: 116, 2018.

21. Pasqualini L, Bu H, Puhr M, Narisu N, Rainer J, Schlick B, Schäfer G, Angelova M, Trajanoski Z, Börno ST, et al: mR-22 and miR-29a are members of the androgen receptor cistrome modulating LAMC1 and Mcl-1 in prostate cancer. Mol Endocrinol 29: 1037-1054, 2015.

22. Wang G, Shen N, Cheng L, Lin J and Li K: Downregulation of miR-22 acts as an unfavorable prognostic biomarker in osteosarcoma. Tumour Biol 36: 7891-7895, 2015.

23. Bersani F,Lingua MF, Morena D, Foglizzo V, Miretti S, Lanzetti L, Carrà G, Morotti A, Ala U, Provero P, et al: Deep sequencing reveals a novel miR-Regulatory network with therapeutic potential in rhabdomyosarcoma. Cancer Res 76: 6095-6106, 2016.

24. Koufaris C, Valbuena GN, Pomyen Y, Tredwell GD, Nevedomskaya E, Lau CH, Yang T, Benito A, Ellis JK and Keun HC: Systematic integration of molecular profiles identifies miR-22 as a regulator of lipid and folate metabolism in breast cancer cells. Oncogene 35: 2766-2776, 2016.

25. Jafarzadeh-Samani Z, Sohrabi S, Shirmohammadi K, Effatpanah H, Yadegarazari R and Saidijam M: Evaluation of miR-22 and miR-20a as diagnostic biomarkers for gastric cancer. Chin Clin Oncol 6: 16, 2017.

26. Fu Q, Liu CJ, Zhang X, Zhai ZS, Wang YZ, Hu MX, Xu XL, Zhang HW and Qin T: Glucocorticoid receptor regulates expression of microRNA-22 and downstream signaling pathway in apoptosis of pancreatic acinar cells. World J Gastroenterol 24: 5120-5130, 2018

27. Sui J, Liu Q, Zhang $\mathrm{H}$ and Kong Y: Deep integrative analysis of microRNA-mRNA regulatory networks for biomarker and target discovery in chondrosarcoma. J Cell Biochem 120: 9631-9638, 2019.

28. Wang D, Guo C, Kong T, Mi G, Li J and Sun Y: Serum miR-22 may be a biomarker for papillary thyroid cancer. Oncol Lett 17: 3355-3361, 2019.

29. Pellatt DF, Stevens JR, Wolff RK, Mullany LE, Herrick JS, Samowitz W and Slattery ML: Expression profiles of miRNA subsets distinguish human colorectal carcinoma and normal colonic mucosa. Clin Transl Gastroenterol 7: e152, 2016.

30. Medarova Z, Pantazopoulos $P$ and Yoo B: Screening of potential miRNA therapeutics for the prevention of multi-drug resistance in cancer cells. Sci Rep 10: 1970, 2020.

31. Garaude J, Kent A, van Rooijen N and Blander JM: Simultaneous targeting of toll- and nod-like receptors induces effective tumor-specific immune responses. Sci Transl Med 4: 120ra116, 2012.

(i) $(-)$ This work is licensed under a Creative Commons Attribution-NonCommercial-NoDerivatives 4.0 International (CC BY-NC-ND 4.0) License. 